\title{
A comprehensive ultrasonographic investigation of the common flexor tendon and medial epicondyle in asymptomatic individuals
}

\author{
Selçuk Parlak ${ }^{1}$ Muhammed Said Beşler ${ }^{1}$, Hamza Özer ${ }^{1}$ Bedriye Başkan² ${ }^{2}$ Uğur Toprak ${ }^{3}$
}

${ }^{1}$ Radiology Department, Ankara Numune Research and Education Hospital, Health Sciences University, Ankara, ${ }^{2}$ Physical Therapy and Rehabilitation Clinic, Ankara Numune Research and Education Hospital, Health Sciences University, Ankara, ${ }^{3}$ Radiology Department, Eskişehir Osmangazi University Faculty of Medicine, Eskişehir, Turkey

\begin{abstract}
Aim: To investigate the common flexor tendon (CFT) thickness and the frequency of ultrasound (US) pathological alterations in its structure in a healthy population and to evaluate if these findings can be correlated with dominant hand, age, gender, body mass index, workload, menopause status. Material and methods: The study population consisted of 111 healthy volunteers. Bilateral CFT thicknesses were measured at $5 \mathrm{~mm}, 10 \mathrm{~mm}$ and $15 \mathrm{~mm}$ distance to medial epicondyle. Tendon echotexture, tears, calcification, enthesophyte, and neovascularization were also evaluated. Results: The CFT was thicker on the dominant side at all three locations $(2.1$ versus $1.9 \mathrm{~mm}$ proximal, 4.0 versus $3.7 \mathrm{~mm}$ in the middle and 7.0 versus 6.6 $\mathrm{mm}$ distal, all $\mathrm{p}<0.05)$. Thickness correlated negatively with postmenopausal duration in all landmarks, except for the nondominant side distal part and positive with body mass index, significantly on the dominant side proximal and middle parts. A statistically significant correlation was found between heavy workers group and tendon thicknesses on the proximal and middle parts of the dominant side and the distal part of the non-dominant sides. US tendinosis was detected in 24/222 tendons, calcification in 11/222 tendons and enthesophyte in 33/222 tendons. The threshold age was 46.5 years for enthesophytes and 51.5 years for calcifications. The prevalence of calcification and enthesophyte tended to be higher in blue-collar workers and on the dominant side. Conclusions: The CFT thickness varies depending on measurement locations, handedness, body mass index, postmenopausal duration and workload. The US signs of tendinosis may be encountered in a symptom-free population. Calcification and enthesophyte tended to be more frequent in blue-collar workers.
\end{abstract}

Keywords: common flexor tendon; medial epicondyle; ultrasound; tendinosis

\section{Introduction}

The flexor-pronator mass is formed by the pronator teres (PT) and the common flexor tendon (CFT). CFT consists of the flexor carpi radialis, palmaris longus,

Received 18.02.2019 Accepted 14.04.2019

Med Ultrason

2019, Vol. 21, No 3, 273-278

Corresponding author: Selçuk Parlak

Radiology Department, Ankara Numune

Research and Education Hospital,

Health Sciences University,

44 Talatpaşa Street, Altındağ,

06230 Ankara, Turkey

E-mail: selcukparlakdr@gmail.com

Phone: +90 5052499563 flexor carpi ulnaris, and flexor digitorum superficialis tendons and is attached to the medial epicondyle of the humerus. This tendon is approximately three centimeters in length, crossing the ulnohumeral joint medially and running parallel to the ulnar collateral ligament, where it serves as a secondary stabilizer. The basic function of CFT is flexion of the hand and wrist and PT mainly pronates the forearm $[1,2]$.

Medial epicondylitis is an overuse tendinopathy due to the chronic repetitive concentric or eccentric loading of the wrist flexors and PT being characterized by insidious medial elbow pain that increases with wrist flexion, forearm pronation and gripping movement. Overall prevalence of medial epicondylitis is $<1 \%$, but it may impair 3.8-8.2\% of patients, depending on occupational 
settings. The population of 40 -to- 60 year-olds has been found to be particularly affected $[1,3,4]$.

Ultrasound (US) is the ideal imaging technique for evaluation of the elbow joint. CFT can be evaluated in detail up to the attachment points on the medial epicondyle (ME). The combination of the superficial positioning of the elbow joint and the high frequency linear US transducer results in high-resolution imaging. Ligaments, muscles, tendons, nerves and osseous structures can be assessed using US [1-5]. Tendinopathy is a general used term to indicate sonographically focal or diffuse hypoechoic areas (tendinosis), partial tear or complete tear [2].

Similar to degenerative joint disease, tendon aging is inevitable over time, generally asymptomatic. Asymptomatic tendinopathy in the rotator cuff and common extensor tendon are well-known entities, proven radiologically $[6,7]$. Imaging findings of tendinosis may be incidentally found in asymptomatic individuals in which US examinations are undertaken for different reasons [7]. Due to the relative low frequency of pathology related to ME comparing with those related to a lateral epicondyle, the US study of this region was done only in a few studies [810]. No study concerning the presence of asymptomatic tendinosis in ME has been published. Thus, the aim of this study was to investigate the relationship between personal variables and asymptomatic tendinosis in CFT.

\section{Material and methods}

This study was approved by the institutional review board. A review of the design of the study was given to all participants and full, written informed consent was obtained. The study group consisted of 111 healthy volunteers admitted to the US department for reasons unrelated to the purpose of the study between January and May 2018. All volunteers were evaluated by the same physical therapy and rehabilitation physician to ensure that they were totally asymptomatic in the ME region. A total of 222 elbows were investigated. The participants were engaged in various professions and jobs, and the study population was classified in terms of workload. Blue-collar workers (74 participants), including laborers, cleaners and farmers, were classified as having a heavy workload. White-collar workers (37 participants), including teachers, students and retirees, were classified as having a light workload. Age, gender, handedness, and weight and height (for the body mass index [BMI] calculation) were recorded for all participants. In addition, menopause status was noted for the female participants.

US examination was performed by an Aplio 500 (Toshiba, Tokyo, Japan) with a $12 \mathrm{MHz}(7-18 \mathrm{MHz})$ linear probe. All US imaging and measurements were undertaken by a musculoskeletal radiologist with 10 years of experience. A standard technique, outlined by the European Society of Musculoskeletal Radiology, was used [11]. For examination of the medial elbow, the patient was asked to lean toward the ipsilateral side with the forearm in forceful external rotation while keeping the elbow extended or slightly flexed, resting on a table (fig 1a). The coronal planes, with the cranial edge of the probe placed over ME, revealed CFT from its long axis [11]. Care was taken to avoid probe compression and prevent incorrect measurements. The tendon thicknesses were measured at regions $5 \mathrm{~mm}$ (proximal part), $10 \mathrm{~mm}$ (middle part), and $15 \mathrm{~mm}$ (distal part) from the ME (fig 1b). Each measurement was performed three times and the average was used for further analysis. Tendon echogenicity, hypoechoic areas within the tendon, tendon rupture, calcification, peritendinous effusion and enthesophyte parameters were investigated in detail. Diffuse or focal hypoechoic areas within the tendon were interpreted as tendinosis. The color-flow signals showing intratendinous hyperemia were evaluated with a combination of color and power Doppler US. To evaluate lowflow vessels, the pulse repetititon frequency was set to the low velocity scale $(100-400 \mathrm{~Hz})$, and color gain was adjusted just below noise level (40-55 dB) to avoid aliasing artifacts.

\section{Statistical analysis}

IBM SPSS Statistics for Windows, Version 20.0 (IBM Corp., Armonk, NY, USA; released 2011) was used for the statistical analysis. The descriptive statistics and parametric outcomes were calculated, with standard deviations. The Kolmogorov-Smirnov test was used to estimate the distribution of the variables, the means and standard deviations to compare the variables, and Student's $t$-test to analyze the continuous variables. The relationships between the CFT thickness and workload, weight, gender, menopause status, duration of menopause, and BMI were examined using Spearman's rank correlation coefficients. Categorical variables were evaluated using a chi-squared test. The area under the estimated receiver operating characteristics (ROC) curve was chosen as the threshold for sensitivity and specificity assessment. The results were evaluated at a $95 \%$ confidence interval, and $\mathrm{p} \leq 0.05$ was accepted as statistically significant.

\section{Results}

The mean age of the participants ( 79 female and 32 male) was $45.8 \pm 15.2$ years. Thirty-two of the female volunteers were in the menopause. The mean BMI was $26.4 \pm 5.5 \mathrm{~kg} / \mathrm{m}^{2}$. CFT was thicker at all three points on the dominant side (Table I). 
Table I. Relationship between dominancy and common flexor tendon thickness (mm)

\begin{tabular}{llll}
\hline & Dominant arm & Non-dominant arm & p value \\
\cline { 2 - 4 } Proximal $(5 \mathrm{~mm})$ & $2.1 \pm 0.4$ & $1.9 \pm 0.4$ & 0.005 \\
Middle $(10 \mathrm{~mm})$ & $4.0 \pm 0.7$ & $3.7 \pm 0.7$ & 0.004 \\
Distal $(15 \mathrm{~mm})$ & $7.0 \pm 1.3$ & $6.6 \pm 1.3$ & 0.018 \\
\hline
\end{tabular}

Table II. Relationship between gender and common flexor tendon thickness

\begin{tabular}{llllll}
\hline \multirow{3}{*}{ Dominant arm } & Gender & n & Proximal & Middle & Distal \\
\cline { 2 - 6 } & Female & 79 & $2 \pm 0.4$ & $3.9 \pm 0.6$ & $6.8 \pm 1.2$ \\
& Male & 32 & $2.2 \pm 0.4$ & $4.3 \pm 0.7$ & $7.6 \pm 1.2$ \\
\multirow{3}{*}{ Non-dominant arm } & p value & & 0.018 & 0.001 & 0.001 \\
& Female & 79 & $1.9 \pm 0.4$ & $3.6 \pm 0.7$ & $6.3 \pm 1.2$ \\
& Male & 32 & $2 \pm 0.5$ & $3.9 \pm 0.8$ & $7.4 \pm 1.3$ \\
\hline
\end{tabular}

$\mathrm{n}$ - number of patients

Table III. Relationship between age, body mass index (BMI), occupational status, postmenopausal duration and common flexor tendon thickness

\begin{tabular}{llllllllll}
\hline Variables & & $\begin{array}{l}\text { Age } \\
\text { (n:111) }\end{array}$ & & $\begin{array}{l}\text { BMI } \\
(\mathbf{n}: 111)\end{array}$ & & \multicolumn{2}{c}{$\begin{array}{l}\text { Occupational status } \\
\text { (n:111) }\end{array}$} & $\begin{array}{l}\text { Postmenopausal dura- } \\
\text { tion (years) (n:32) }\end{array}$ \\
\cline { 3 - 10 } & & $\mathrm{r}$ & $\mathrm{p}$ & $\mathrm{r}$ & $\mathrm{p}$ & $\mathrm{r}$ & $\mathrm{p}$ & $\mathrm{r}$ & $\mathrm{p}$ \\
\hline Dominant & Proximal & -0.01 & 0.944 & 0.33 & $<0.001$ & 0.204 & 0.045 & -0.56 & 0.001 \\
arm & Middle & 0.03 & 0.779 & 0.23 & 0.017 & 0.223 & 0.028 & -0.56 & 0.001 \\
& Distal & 0.03 & 0.061 & 0.15 & 0.113 & 0.057 & 0.579 & -0.59 & $<0.001$ \\
Non-dominant & Proximal & -0.01 & 0.972 & 0.16 & 0.088 & 0.086 & 0.421 & -0.43 & 0.013 \\
arm & Middle & -0.05 & 0.598 & 0.11 & 0.259 & 0.153 & 0.108 & -0.39 & 0.028 \\
& Distal & -0.07 & 0.463 & 0.07 & 0.492 & 0.205 & 0.049 & -0.27 & 0.128 \\
\hline
\end{tabular}

$\mathrm{n}$ - number of patients

Table IV. Distribution of the ultrasound findings

\begin{tabular}{llll}
\hline & Dominant & Non-dominant & Affected/total elbows \\
\cline { 2 - 3 } Tendinosis & $14(6.3)$ & $10(4.5)$ & $24 / 222$ \\
Calcification & $11(4.9)$ & $0(0)$ & $11 / 222$ \\
Enthesophyte & $19(8.5)$ & $14(6.3)$ & $33 / 222$ \\
\hline
\end{tabular}

The results are expressed as number (\%)

Males had thicker tendons for all landmarks, except for the non-dominant arm proximal section (Table II). The results of CFT thickness showed that BMI was slightly positively correlated with the proximal and middle sections of dominant arms. A moderately negative correlation between postmenopausal duration and CFT thickness was seen in all landmarks, other than the non-dominant side distal part. Correlation with age has no statistically significance. Tendon thicknesses were greater on the proximal and middle parts of the dominant side and the distal part of the non-dominant sides in heavy duty workers, with statistical significance (Table III). No correlation was found between CFT thickness and menopause status $(\mathrm{p}>0.05)$.

Tendinosis was seen in 15 blue-collar workers in $24 / 222$ tendons $(10.8 \%$ ), bilateral in 9 cases (fig 1c). Calcification in $11 / 222$ tendons $(4.9 \%)$ and enthesophyte in 33/222 tendons (14.8\%) were seen (fig 1d). Although enthesophyte and calcification were seen more frequently on dominant sides, the arm dominancy effect on enthesophyte or calcification formation was not found to be statistically significant ( $p>0.05$ ) (Table IV). The enthesophyte and calcification rates were higher in blue-collar workers than white-collar workers; however, these were not significant (all $\mathrm{p}>0.05$ ). A ROC curve analysis was performed for calcification (fig 2a) and enthesophytes (fig $2 \mathrm{~b}$ ). The enthesophyte and calcification prevalence was high above the threshold ages. The threshold age was estimated as 46.5 years for enthesophytes (sensitivity $97.4 \%$ and specificity $65.8 \%$ ) and 51.5 years for calcifications (sensitivity $87.5 \%$ and specificity $65 \%$ ). None of the participants had tendon rupture, effusion and neovascularization on gray-scale US or Doppler US. 


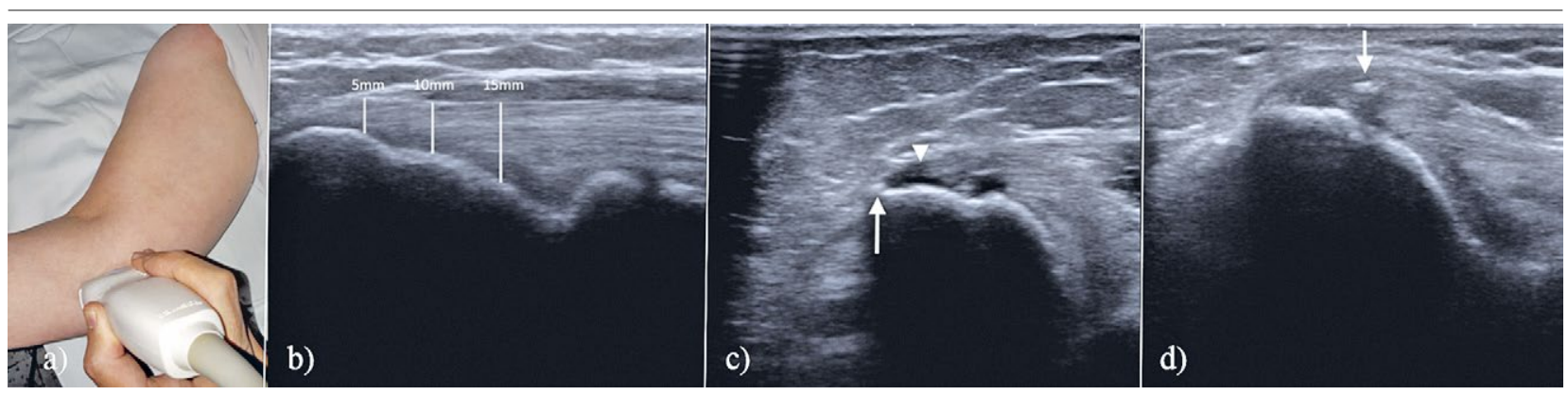

Fig 1. a) Longitudinal probe position for the common flexor tendon examination; b) longitudinal sonographic view of the common flexor tendon and measurement points at 5-10-15 mm distance to the medial epicondyle (lines); c) enthesophyte (arrow) in the medial epicondyle and focal hypoechoic area (arrowhead) compatible with tendinosis in the common flexor tendon proximal region. Note the irregularity in the bone cortex; d) intratendinous calcification (arrow) in the common flexor tendon in an asymptomatic participant.

\section{Discussions}

In this study, US characteristics of CFT were investigated in a general asymptomatic population with no medial epicondylitis history or symptoms. In a classical approach, common extensor tendon measurements are carried out at its thickest part (musculotendinous junction) $[12,13]$; however, some studies have used different locations $[7,14]$. No method for CFT thickness measurement was described and US studies of ME focused on intratendinous and bony changes [8-10]. In this study, measurements were undertaken at three different locations, which helped to provide more comparable data. The results showed that the tendon tended to be thinner at the proximal part, gradually thickening toward the distal portion.

Klauser et al performed a study on cadavers and decribed in detail the sonoelastographic features of CFT in comparison with histology [8]. Park et al intensively investigated 46 elbows in symptomatic and healthy populations and found that US is an accurate imaging technique in detection of clinical medial epicondylitis [9]. The comprehensive thickness measurements of CFT at different locations, workload classifications, menopause situation and the totally asymptomatic population are the basic differences of our study. We found the CFT to be thicker on the dominant side at all three measurement locations and, apart from the proximal part of the non-dominant arm, males had thicker tendons than females. Shiri et al noted that medial epicondylitis incidence is related to working years and obesity [15]. In our study, BMI was positively correlated with the proximal and middle sections of the dominant side, but this relationship was not observed on the non-dominant side nor on the distal part of the dominant side. The age of participants was found to be unrelated with tendon thickness. Ustuner et al reported that CET thickened with age and BMI in a linear fashion and males had thicker tendons than females only in the humeral region [7].

The menopause process results in withdrawal of the estrogen hormone. The reduction of blood estrogen level is associated with a decrease in collagen synthesis, fiber diameter and density and an increase in degradation in tendon tissue [16-18]. In this study, a negative correlation between postmenopausal duration and CFT thickness was detected in all landmarks, except for the distal part of the non-dominant side.

We found asymptomatic US tendinosis in 24/222 tendons $(10.8 \%$.) Park et al found in CFT focal hypoechoic areas in $4 \%$ of the healthy participants [9]. The lower tendinosis ratio compared to our study is most likely caused by sampling difference but we can not give supplementary data due to the fact that the authors did not describe the control group features [9]. We found all cases with tendinosis in the blue-collar workers group and this could be related to the increased repetitive microtrauma effect. It should also be kept in mind that gray-scale US changes are known to exist in asymptomatic patients and reported to persist after the resolution of complaints or
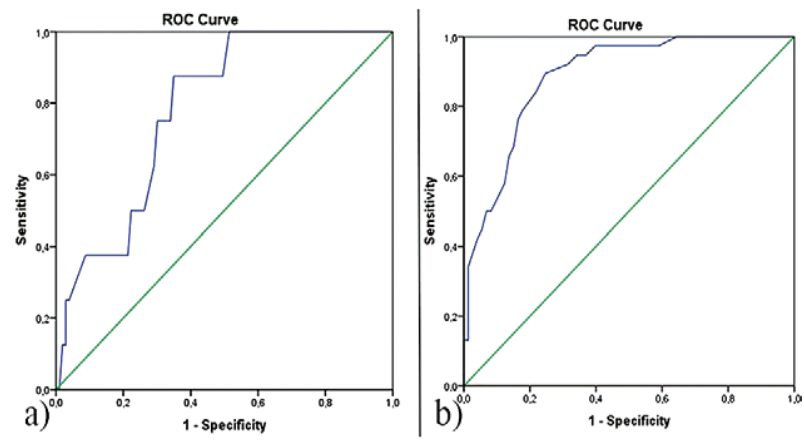

Fig 2. a) ROC curve analysis comparing calcification in relation to age; b) ROC curve analysis comparing the bony changes in relation to age. 
even after treatment $[12,19,20]$. Tears, joint effusions and neovascularization represent active tendinopathy, in addition to which neovascularity is presumed to be linked with pain [19]. A previous study found increased vascularity in $19 \%$ of painful medial epicondylitis cases [9]. We did not observe pathological vascularization in CFT probably due to the lack of symptoms, especially pain, in our group. No ruptures or joint effusions were seen either.

Park et al found intratendinous calcification in 24\% of clinical medial epicondylitis cases, but none in asymptomatic participants [9]. We found asymptomatic calcification in $4.9 \%$ of subjects. Jaen-Diaz et al observed that incidence of bony spurs increases with age [12] and Krogh et al suggested that loading and bony spur formation were related based on arm dominancy [14]. We found that both calcification and enthesophyte formation increased with aging and tended to be more frequent on the dominant sides, but these findings have no statistically significance. In a similar manner, enthesophyte and calcification were recorded at a higher rate in blue-collar workers, even though this relation was not statistically significant. Increases in bony changes (spurs or enthesophytes) are also thought to be risk factors for symptomatic tendinopathies [19-21]. For this reason, the followup of blue-collar workers, decreasing microtraumas and investigating the impact of working in the wrong position could be helpful in terms of preventive medicine and public health.

There are some limitations of our study. First is the lack of comparison between symptomatic and asymptomatic individuals. Also, no longitudinal follow-up of our subject was realized in order to evaluate the predictive power of the US pathological findings for development of the symptomatic disease. Intra- and inter-observer agreement concerning the US examinations were not evaluated. Finally, no correlations with the type of repetitive movement of the healthy subjects and physical activities was realized.

In conclusion, to the best of our knowledge, this study is the most comprehensive investigation of ME as it relates to tendon thickness, age, gender, bony changes and tendon appearance, as well as menopause and workload. Arm dominancy, gender, postmenopausal duration and BMI have an obvious relationship with tendon thickness. Calcification and enthesophyte prevalence tended to be higher in blue-collar workers and on the dominant side, even though these were not statistically significant.

Conflict of interest: none

\section{References}

1. Amin NH, Kumar NS, Schickendantz MS. Medial epicondylitis: evaluation and management. J Am Acad Orthop Surg 2015;23:348-355.

2. Konin GP, Nazarian LN, Walz DM. US of the elbow: indications, technique, normal anatomy, and pathologic conditions. Radiographics 2013;33:E125-E147.

3. Donaldson O, Vannet N, Gosens T, Kulkarni R. Tendinopathies Around the Elbow Part 2: Medial Elbow, Distal Biceps and Triceps Tendinopathies. Shoulder Elbow 2014;6:47-56.

4. Kiel J, Kaiser K. Golfers Elbow. StatPearls Publishing, 2018.

5. De Maeseneer M, Brigido MK, Antic M, et al. Ultrasound of the elbow with emphasis on detailed assessment of ligaments, tendons, and nerves. Eur J Radiol 2015;84:671-681.

6. Keener JD, Steger-May K, Stobbs G, Yamaguchi K. Asymptomatic rotator cuff tears: patient demographics and baseline shoulder function. J Shoulder Elbow Surg 2010;19:1191-1198.

7. Ustuner E, Toprak U, Baskan B, Oztuna D. Sonographic examination of the common extensor tendon of the forearm at three different locations in the normal asymptomatic population. Surg Radiol Anat 2013;35:547-552.

8. 8. Klauser AS, Pamminger MJ, Halpern EJ, et al. Sonoelastography of the Common Flexor Tendon of the Elbow with Histologic Agreement: A Cadaveric Study. Radiology 2017;283:486-491.

9. Park GY, Lee SM, Lee MY. Diagnostic value of ultrasonography for clinical medial epicondylitis. Arch Phys Med Rehabil 2008;89:738-742.

10. Suresh SP, Ali KE, Jones H, Connell DA. Medial epicondylitis: is ultrasound guided autologous blood injection an effective treatment? Br J Sports Med 2006;40:935-939.

11. Beggs I, Bianchi S, Bueno A, et al. Musculoskeletal technical guidelines. Part 2. Elbow. European Society of Musculoskeletal Radiology. Available at: http://www.essr.org/ html/img/pool/elbow.pdf. Accessed in 17 Feb 2019.

12. Jaen-Diaz JI, Cerezo-Lopez E, Lopez-de Castro F, et al. Sonographic findings for the common extensor tendon of the elbow in the general population. J Ultrasound Med 2010;29:1717-1724.

13. Lee MH, Cha JG, Jin W, et al. Utility of sonographic measurement of the common tensor tendon in patients with lateral epicondylitis. AJR Am J Roentgenol 2011;196:13631367.

14. Krogh TP, Fredberg U, Ammitzbol C, Ellingsen T. Ultrasonographic Characteristics of the Common Extensor Tendon of the Elbow in Asymptomatic Individuals: Thickness, Color Doppler Activity, and Bony Spurs. Orthop J Sports Med 2017;5:2325967117704186.

15. Shiri R, Viikari-Juntura E, Varonen H, Heliovaara M. Prevalence and determinants of lateral and medial epicondylitis: a population study. Am J Epidemiol 2006;164:1065-1074.

16. Koivunen-Niemela T, Parkkola K. Anatomy of the Achilles tendon (tendo calcaneus) with respect to tendon thickness measurements. Surg Radiol Anat 1995;17:263-268. 
17. Frizziero A, Vittadini F, Gasparre G, Masiero S. Impact of oestrogen deficiency and aging on tendon: concise review. Muscles Ligaments Tendons J 2014;4:324328.

18. Leblanc DR, Schneider M, Angele P, Vollmer G, Docheva D. The effect of estrogen on tendon and ligament metabolism and function. J Steroid Biochem Mol Biol 2017; 172:106-116.

19. du Toit C, Stieler M, Saunders R, Bisset L, Vicenzino B. Diagnostic accuracy of power Doppler ultrasound in patients with chronic tennis elbow. Br J Sports Med 2008;42:872876.

20. Zeisig E, Fahlstrom M, Ohberg L, Alfredson H. A twoyear sonographic follow-up after intratendinous injection therapy in patients with tennis elbow. Br J Sports Med 2010;44:584-587.

21. Struijs PA, Spruyt M, Assendelft WJ, van Dijk CN. The predictive value of diagnostic sonography for the effectiveness of conservative treatment of tennis elbow. AJR Am J Roentgenol 2005;185:1113-1118. 